\title{
X-ray reflectivity method for the characterisation of InGaN/GaN quantum well interface
}

\author{
Fabien Massabuau*, Nicolas Piot, Martin Frentrup, Xiuze Wang, Quentin Avenas, Menno Kappers, Colin \\ Humphreys, Rachel Oliver.
}

Department of Materials Science and Metallurgy, University of Cambridge, 27 Charles Babbage Road, Cambridge CB3 0FS, United Kingdom

Received XXXX, revised XXXX, accepted XXXX

Published online XXXX

Key words: III-nitrides, X-ray reflectivity, quantum well, interface.

* Corresponding author: e-mail fm350@cam.ac.uk, Phone: +44 1223 334044, Fax: +44 1223334437

A method to characterise the interface of InGaN/GaN quantum wells by X-ray reflectivity is presented. The interface roughness can be obtained from the ratio of diffuse to specular scatterings obtained on a transverse $\omega$-scan. Rotation around the azimuthal $\phi$ angle allows for information about the directionality of the roughening mechanisms to be obtained. The method allows for quick identification of the presence or absence of gross well width fluctuations in the quantum well, providing that the interface is chemically sharp. When the interface exhibits chemical grading, compositional fluctuations across the terraced structure of the quantum well surface lead to aggravated roughness as the barrier is grown, which may be misinterpreted as gross well width fluctuations.

This method carries promises for complementing analysis by transmission electron microscopy as it is nondestructive, fast and allows multi-directional characterisation of the roughness. It would therefore be particularly useful to detect process deviation in a production line, where prior knowledge of the sample is already available.
1 Introduction InGaN/GaN quantum wells (QWs) constitute the active region of efficient violet, blue and green light emitting diodes (LEDs) [1]. In the context of improving the performance of such devices, an intense research effort has been dedicated in the recent years to QW design. Most of them involve tailoring the InGaN/GaN interface, which fall into two main categories: topological roughness and interfacial grading. Topological roughness here implies a sharp change in composition, but a rough interface. In InGaN/GaN QW structures, the thickness of the QWs is never perfectly uniform and results in either monolayer variations [2,3], or in gross-well width fluctuations (GWWFs) [3-6]. The latter configuration is generally obtained when the QW is directly exposed to a high temperature during growth. It results in the formation of InGaN strips separated by troughs, and dislocations are found to preferentially thread towards the surface through the resulting gaps in the QW [4], thus reducing the non- radiative recombination in the QW [5]. Interfacial grading corresponds to an interface that is topographically flat but exhibits a gradient in composition. This type of interface has been reported to impact the internal electric field [7, 8], the electron-hole wavefunction overlap [7-11] or the Auger recombination rate $[12,13]$. It is therefore important to be able to characterise the QW interface. However until now, this was done using transmission electron microscopy (TEM) $[5,10,14-16]$ or atom probe tomography (APT) [2, 6]. However these methods are time-consuming, destructive, and can be experimentally challenging when beam damage of the QWs $[17,18]$ is taken into consideration.

$\mathrm{X}$-ray reflectivity (XRR) is a powerful technique for thin film characterisation [19] which is generally employed to determine the thickness of QWs [20-23]. However it can also be used to extract information relative to the interface of thin layers [19,24-26]. However to our knowledge, this method is very rarely employed in the Nitride community 
despite its potential application for accurate QW interface characterisation. In this study, we aim to provide a methodology based on XRR to allow for a characterisation of QW interface which is non-destructive, fast, low cost and easily accessible (in terms of equipment requirement and user's expertise) compared to TEM or APT.

2 Basics of X-ray reflectivity XRR is based on the reflection of low angle incident X-rays by interfaces between materials with different refractive indices. The quality of the interface hence plays an important role in determining the reflected intensity. A key parameter to describe the interface is the interface width, which falls into two main categories: grading and roughness. A graded interface corresponds to a flat interface which exhibits a gradient in composition, while a rough interface relates to an undulating interface with a chemically sharp boundary (See Figure 1). The interface width can generally be obtained from a specular scan (i.e. a low angle symmetric $\omega-2 \theta$ scan), as it relates to the damping of the signal. However this method does not allow one to distinguish between the two types of interface broadening mechanisms and a transverse scan (i.e. an $\omega$ scan) is required. Given that the gradual change in refractive index of a graded interface scatters the X-ray out-of-phase, interfacial grading solely results in a loss of intensity. On the other hand, in the case of a rough interface, the chemically sharp but locally inclined interface scatters the incident X-rays with an offset relative to the specular scattering position, called diffuse scattering (See Figure 1). Figure 2 shows a typical transverse scan from a QW structure highlighting the parts of the signal corresponding to specular and diffuse scattering. As can be seen, the transverse scan consists of a main, central, spec-
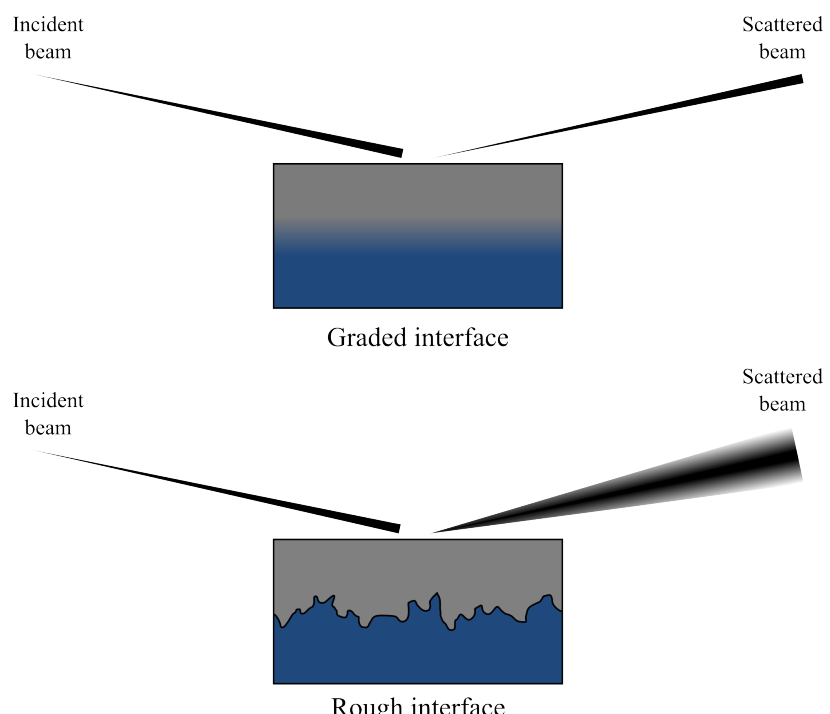

Figure 1 Schematic illustrating the two interface broadening mechanisms, grading (top) and roughness (bottom), and how these affect the scattered beam.

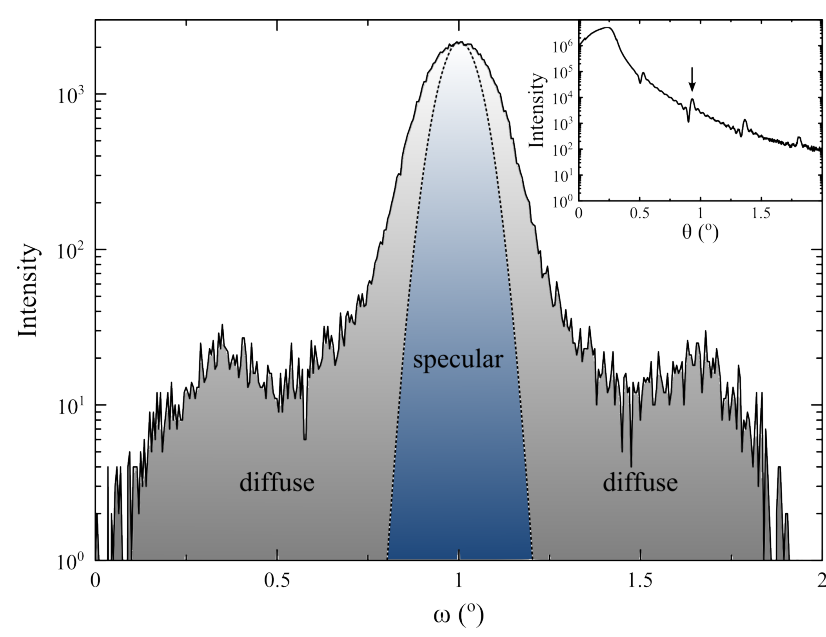

Figure 2 Transverse scan from a QW structure highlighting the specular and diffuse scattering from the sample. In inset the specular $\omega-2 \theta$ scan of the structure. The arrow indicate the $\omega$ and $2 \theta$ positions at which the transverse scan is taken.

ular peak, and a diffuse scattering signal which appears as high angle wings and as broadening of the specular peak.

A formalism under the first order Born approximation has been developed to simulate off-specular scattering from rough surfaces [27]. This approach has been successfully used in the past to determine the interface quality of materials observed under synchrotron radiation [24, 27]. However this method is computationally demanding and the use of synchrotron radiation undermines our initial purpose to provide a quick, low-cost and accessible alternative to TEM and APT. In this study, we will use a simplification of the original formalism proposed by Bowen and Tanner [19] which we apply to scans taken on a laboratory X-ray diffractometer. From this simplification, the root mean square (RMS) roughness $\sigma$ of the interface can be readily estimated from the ratio of the diffuse to specular scattering intensities according to the formula:

$$
\frac{I_{\text {diff }}}{I_{\text {spec }}}=e^{Q_{z}^{2} \sigma^{2}}-1
$$

where $I_{\text {diff }}$ and $I_{\text {spec }}$ are the (integrated) intensities which have been diffusely and specularly scattered, respectively, $Q_{z}=\frac{4 \pi}{\lambda} \sin \theta$, with $\lambda$ the wavelength of the X-ray radiation, and $\theta$ the semi-angle between the incident beam and reflected beam. In a structure with multiple interfaces, the roughness obtained from this method will be an average value of the roughness of each interface.

\section{Experimental methods}

3.1 Sample details Six ten-period InGaN/GaN QW structures were grown by metal-organic vapour phase epitaxy (MOVPE) in a Thomas Swan $6 \times 2$ inch showerhead reactor. Trimethylgallium (TMG), trimethylindium (TMI) and ammonia $\left(\mathrm{NH}_{3}\right)$ were used as precursors for $\mathrm{Ga}$, In 

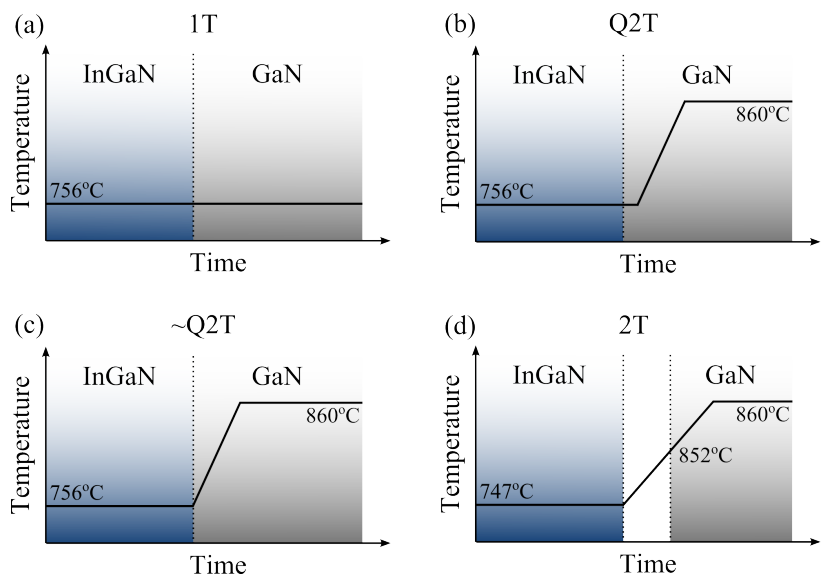

Figure 3 Schematic showing the temperature profile during the growth of the $\mathrm{GaN}$ barrier for the (a) 1T, (b) Q2T, (c) Q2T and (d) $2 \mathrm{~T}$ growth methods.

and $\mathrm{N}$, respectively. Hydrogen $\left(\mathrm{H}_{2}\right)$ was used as the carrier gas for $\mathrm{GaN}$ buffer layer growth and nitrogen $\left(\mathrm{N}_{2}\right)$ as the carrier gas for InGaN/GaN QW growth. Pseudosubstrates consisting of $c a .5 \mu \mathrm{m}$ of $\mathrm{GaN}$ (of which $2 \mu \mathrm{m}$ undoped and $3 \mu \mathrm{m}$ Si-doped to $5 \times 10^{18} \mathrm{~cm}^{-3}$ ) grown on c-plane sapphire with a miscut of $(0.25 \pm 0.1)^{\circ}$ towards $(11 \overline{2} 0)$ were employed. Four different growth techniques which we refer to as 1T (one temperature), Q2T (quasi two temperature), $\sim$ Q2T (almost quasi two temperature) and 2T (two temperature) were used for the growth of the $\mathrm{GaN}$ barrier (See Figure 3). The temperatures cited here are those of the susceptor, on which the wafers are placed during growth, as measured by emissivity corrected pyrometry (from Laytec). For convenience the samples will be directly referred to by their growth techniques.

For sample 1T, following the growth of a nominally 2.5 $\mathrm{nm}$ InGaN QW at $756^{\circ} \mathrm{C}$, the entire ca. $7.5 \mathrm{~nm}$ GaN barrier is grown at the same temperature. For sample Q2T, a $c a .1 \mathrm{~nm}$ thin protective GaN cap layer is grown on top of the QW before ramping the temperature to $860^{\circ} \mathrm{C}$, during which an additional $1 \mathrm{~nm}$ of $\mathrm{GaN}$ is being deposited. The growth of $\mathrm{GaN}$ goes on whilst the temperature is maintained at $860^{\circ} \mathrm{C}$ until a total of $c a .7 .5 \mathrm{~nm}$ of $\mathrm{GaN}$ has been deposited. For sample $\sim \mathrm{Q} 2 \mathrm{~T}$, the temperature is ramped to $860^{\circ} \mathrm{C}$ right after the $\mathrm{InGaN} \mathrm{QW}$ is grown at $756^{\circ} \mathrm{C}$. Ca. $1 \mathrm{~nm}$ of $\mathrm{GaN}$ is grown during the temperature ramp and the remaining $\mathrm{GaN}$ is grown at $860^{\circ} \mathrm{C}$. For sample $2 \mathrm{~T}$, to compensate any loss of indium during the temperature ramp, the QW is grown at $747^{\circ} \mathrm{C}$, and the temperature is increased directly after the $\mathrm{InGaN}$ is grown. The growth of the barrier starts when the temperature has reached $852^{\circ} \mathrm{C}$ to settle at $860^{\circ} \mathrm{C}$.

From a previous study which investigated these samples by high-resolution TEM, it was found that 1T, Q2T and $\sim$ Q2T growth methods lead to a $\mathrm{QW}$ top interface which is flat but with a grading decreasing from $1 \mathrm{~T}$ to $\mathrm{Q} 2 \mathrm{~T}$ and $\sim \mathrm{Q} 2 \mathrm{~T}$, and that the growth by $2 \mathrm{~T}$ method results in a rough interface with almost no grading [10]. The roughness induced by the $2 \mathrm{~T}$ method is predominantly due to the presence of GWWFs, which are used here as interface roughening mechanism. In a previous study we reported that the size and spacing of the GWWFs can be controlled to some extent by changing the misorientation of the sapphire substrate [16]. To investigate a range of interface roughnesses, we investigated three samples grown by $2 \mathrm{~T}$ method, with substrate miscut of $0^{\circ}, 0.25^{\circ}$ and $0.5^{\circ}$ $\left( \pm 0.1^{\circ}\right)$ towards $(11 \overline{2} 0)$. (The actual values of the misorientation were found by X-ray diffraction to be very close to the nominal values given by the manufacturer [16].) We will refer to these samples as $2 \mathrm{~T} 0^{\circ}, 2 \mathrm{~T} 0.25^{\circ}$ and $2 \mathrm{~T} 0.5^{\circ}$, respectively.

Finally, in order to simulate the morphology of the QWs, four additional samples were grown. They consist of a nominally $2.5 \mathrm{~nm}$ thin layer of InGaN with no $\mathrm{GaN}$ capping layer. To simulate the growth by $1 \mathrm{~T}, \mathrm{Q} 2 \mathrm{~T}$ and $\sim$ Q2T the growth temperature was quickly decreased right at the end of the growth of the InGaN layer. To simulate the growth by $2 \mathrm{~T}$, following the growth of the InGaN layer on three misoriented substrates similar to the QW samples, the temperature was ramped-up to $860^{\circ} \mathrm{C}$ for $90 \mathrm{~s}$ before cooling down the sample as quickly as possible. This last method has been employed in the past and was found to give a reliable insight into the formation of GWWFs by the 2T method [16].

3.2 AFM Atomic force microscopy (AFM) was performed on a Veeco Dimension 3100 in tapping mode to obtain the RMS roughness of the samples, from $1 \mu \mathrm{m} \times 1 \mu \mathrm{m}$ scans. In the previous section we mentioned that the interfacial roughness obtained from Equation 1 will be the average roughness of all the interfaces present in the sample. While the QW samples can be used to access the morphology of the bottom interface of the QWs (i.e. it is the topography of the GaN surface on top of which a QW would be grown) the thin InGaN layers were deliberately designed to access the topography of the top interface of the QWs. In the case of the QW samples, as we will see in section 4.1, the surface is pinned with defects - V-pits and trench defects - which locally alter the surface morphology. In these samples the roughness is extracted by excluding such defects because these affect the interface differently across the QW stack (i.e. their effect is negligible on the QW located at the bottom of the QW stack and increases at each subsequent QW) making their effect difficult to predict.

3.3 XRR XRR was performed on a Philips X'pert MRD diffractometer with a $\mathrm{Cu}$ target as X-ray source $\left(\lambda_{C u K_{\alpha 1}}=1.5405974 \AA[28]\right)$ followed by a 4 mm mask, a parabolic mirror, a $1 / 32^{\circ}$ divergence slit, an additional beam mask, a beam knife positioned to cut $30 \%$ to $40 \%$ of the signal and a $0.2 \mathrm{~mm}$ receiving slit. The sample was positioned on the stage with the $[11 \overline{2} 0]$ direction perpendicular to the scattering plane. As mentioned in the X-ray basics section, the interface roughness affects the broadening of the main peak in the tranverse scan. However beam 

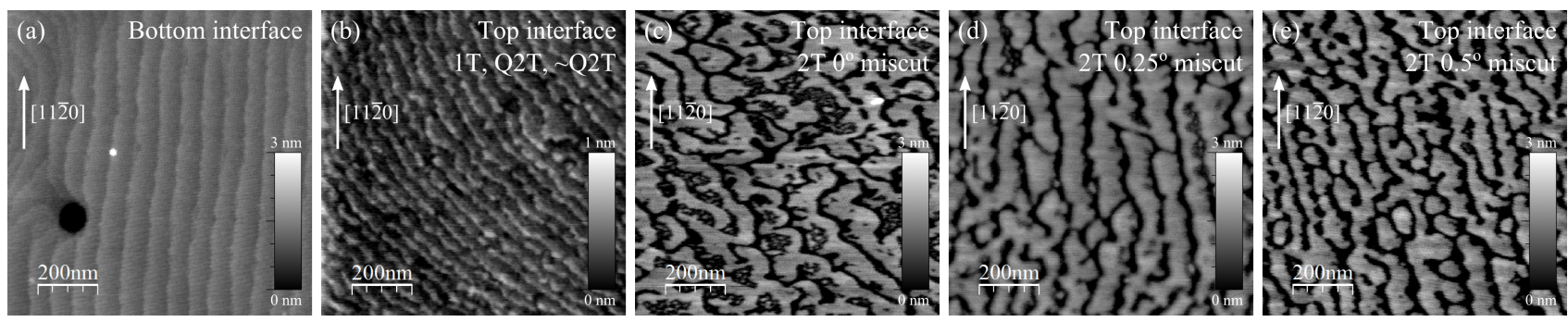

Figure $41 \mu \mathrm{m} \times 1 \mu \mathrm{m}$ AFM scans of sample 1T (a), 1T/Q2T/ Q2T-simulated InGaN thin layer (b), 2T-simulated InGaN thin layer with $0^{\circ}(\mathrm{c}), 0.25^{\circ}$ (d) and $0.5^{\circ}$ (e) substrate misorientation. These scans provide insight into the morphology of the bottom interface of the QWs (a) and top interface of the QWs in samples 1T, Q2T, Q2T (b) and 2T (c)-(e).

divergence and wafer curvature broaden the peak further which, if not considered in the experimental set-up, will lead to erroneous results. The purpose of the narrow divergence slit, the two masks and the beam knife we employed is indeed to limit the illuminated area on the sample and therefore to limit this undesirable broadening.

For each sample, a transverse scans was recorded at the position of the second main oscillation peak on the specular $\omega-2 \theta$ scan, as illustrated in inset of Figure 2 (which corresponds here to an angular position of $2 \theta \sim 2^{\circ}$ ). The main reason for this is that the first order Born approximation, from which Equation 1 is based, is valid only "far" from the critical angle (i.e. $\theta \sim 0.3^{\circ}$ ). The second reason is that this position has locally higher intensity compared to higher angular positions, hence resulting in a higher signal to noise ratio in the transverse scan. The transverse scan was then fitted using a Gaussian fit to extract the specular intensity. Generally the specular intensity is obtained by comparing a specular scan to a near-to-specular scan (i.e. specular scan with a slight offset in $\omega$ ). However this approach is valid for synchrotron radiation [24,27], i.e. when the specular peak is sufficiently narrow and intense. In a table top X-ray diffractometer this approach cannot be applied due to the wider central peak in the transverse scan. Given that the width of the specular peak should be a characteristic of the diffractometer set-up only, and not of the sample, it is expected to be a constant across the entire set of samples. The full width at half maximum of the Gaussian specular fit was thus arbitrarily set to $0.10^{\circ}$. It should be noted that choosing another value for the width of the fit only offsets the RMS roughness of each sample without affecting how the samples compare to each other. Hence, using the notations of Equation 1, $I_{\text {spec }}$ is obtained by the area under the Gaussian specular fit, and $I_{\text {diff }}$ by the difference between the area under the experimental curve and $I_{\text {spec }}$.

\section{Results and Discussion}

4.1 Roughness measurements Figure 4(a) shows the typical morphology of the bottom interface of the QWs. The morphology of the bottom interface exhibits a terraced structure, with atomic layer steps which are gener- ally oriented parallel to the $[11 \overline{2} 0]$ direction, as expected from the substrate miscut direction $[16,29]$. The surface is also affected by defects, V-pits [30] and trench defects [31], which result in inclined facets relative to the growth plane and also pins the atomic steps, i.e. causes the atomic steps to deviate from their main $[11 \overline{2} 0]$ orientation. Figure 4(b)-(e) shows the topography of the InGaN thin layers representative of the top interface of samples $1 \mathrm{~T}, \mathrm{Q} 2 \mathrm{~T}$ and $\sim$ Q2T (b), and samples $2 \mathrm{~T}$ with $0^{\circ}(\mathrm{c}), 0.25^{\circ}(\mathrm{d})$ and $0.5^{\circ}$ (e) substrate miscut. Whilst a terraced structure is clearly observable in the latter, an InGaN strip structure separated by troughs is formed when the InGaN layer is exposed to a high temperature, in line with previous reports [16,29]. Akin to the atomic steps of the GaN underneath it, the In$\mathrm{GaN}$ atomic steps in Figure 5(b) and strips in Figure 5(d)(e) are predominantly oriented parallel to the $[11 \overline{2} 0]$ direction when the substrate has a miscut. As reported earlier, the size and spacing between the strips is reduced when the substrate miscut increases, which is the mechanism we used here to access a range of interface roughnesses.

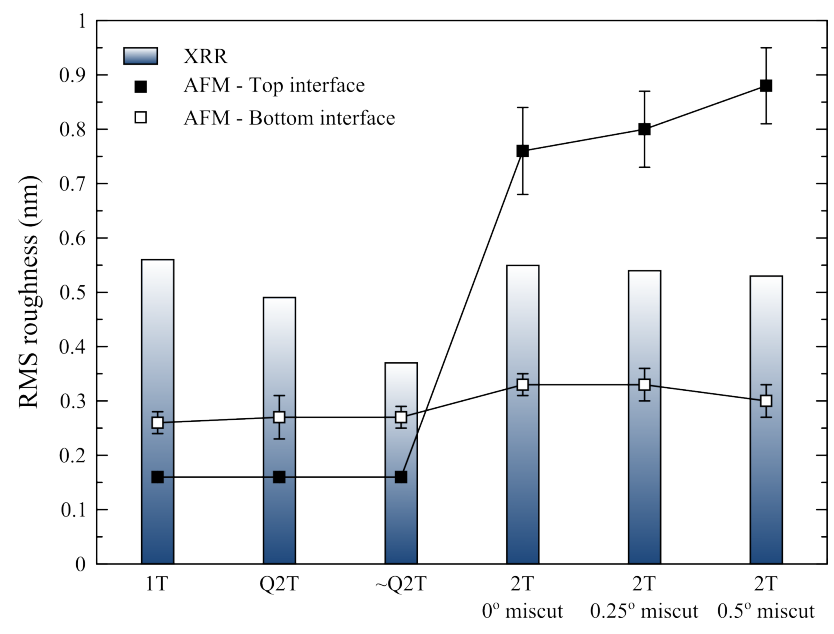

Figure 5 Summary of the interface roughness obtained by AFM and XRR for each sample. (Here the $[11 \overline{2} 0]$ direction of the sample was perpendicular to the scattering plane.) 
Figure 5 summarises the roughness measurements performed on each sample by AFM as well as the results obtained by XRR. The AFM measurements are in line with the observations made based on Figure 4, whereby the bottom surface of the QW exhibits a low roughness (due to the terraced structure) similar across the set of samples, and the top interface roughness is representative of the presence or absence of GWWFs. The GWWFs in the 2T-grown samples is clearly evidenced by the increased roughness compared to samples 1T, Q2T and $\sim$ Q2T. There is a fairly good agreement between the XRR data and the AFM measurements for sample $\sim$ Q2T and the three 2T-grown samples. We can see that the samples exhibiting GWWFs have a greater roughness compared to sample $\sim$ Q2T (It should be kept in mind that the roughness obtained by XRR should be an average of the top and bottom interface roughnesses.). However it does not seem that the XRR approach is able to detect the change in roughness between the 2T-grown samples grown on various substrate miscuts, this point will be further detailed in the next section.

The results obtained for samples $1 \mathrm{~T}$ and $\mathrm{Q} 2 \mathrm{~T}$, and to some extent $\sim \mathrm{Q} 2 \mathrm{~T}$ as well, do not follow our expectations from AFM. Interestingly these samples have been shown by TEM to exhibit incorporation of indium into the GaN barrier [10]. In theory this should be directly interpreted as interface grading and therefore should not affect the roughness measurement obtained from Equation 1. However we see here that the roughness determined by XRR decreases from sample $1 \mathrm{~T}$ to $\mathrm{Q} 2 \mathrm{~T}$ and $\sim \mathrm{Q} 2 \mathrm{~T}$. Similarly, based on [10], the indium tail, interpretable as the width of graded interface, decreases from sample $1 \mathrm{~T}$ to Q2T and $\sim$ Q2T. A potential reason for the surprisingly high roughness measured by XRR is that the indium incorporation reported in this study is not uniform across the growth plane, i.e. the isoconcentration surface is not flat. According to previous reports which investigated the formation of GWWFs, the terraced structure of the GaN barrier underneath a QW affected the indium incorporation in the QW grown above it $[16,29]$. It was suggested that the InGaN material grown on the step edges contained more indium than the InGaN material grown on terraces. Following this reasoning, this implies that in the terraced structure of the InGaN top interface (as pictured in Figure 4(b)), the step edges are richer in indium than the terraces (this could presumably be understood in terms of different properties of the indium surfactant layer at the step edge compared to the terrace). Hence when the GaN barrier is grown above the $\mathrm{QW}$, the magnitude of the incorporation of indium in the barrier at the step edge will be higher than on the terrace, potentially leading to an isoconcentration surface rougher than the terraced structure in Figure 4(b). Given that TEM provides a picture of the material properties over the entire thickness of the TEM foil, this information may have been lost due to projection effects, or could have easily been mistakenly attributed to an artefact due to electron beam damage.

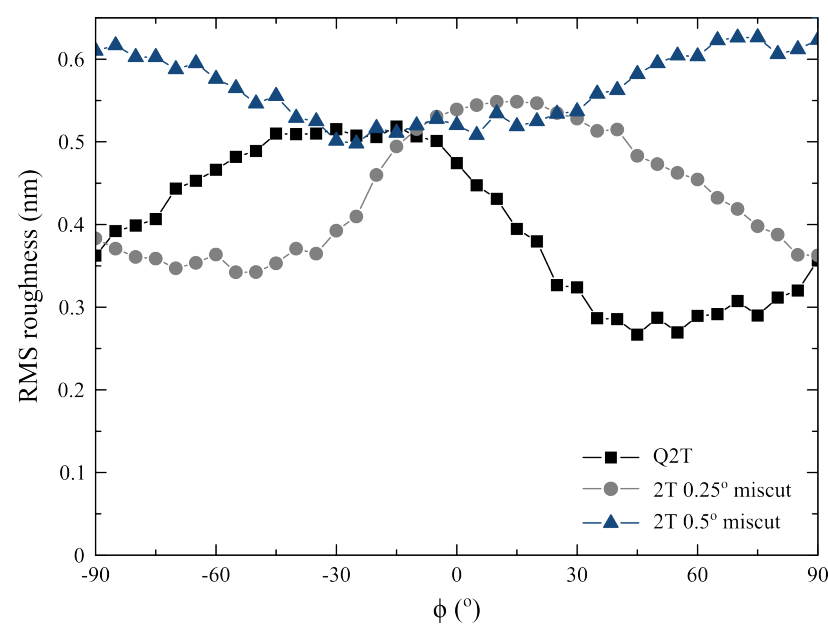

Figure 6 RMS roughness obtained by XRR for different azimuthal angles $\phi$, with $\phi=0^{\circ}$ corresponding to the [1120] direction of the sample perpendicular to the scattering plane.

It should be noted that we did not consider the impact of V-pits and trench defects as a potential explanation for the high roughness of samples 1T, Q2T and $\sim$ Q2T. Although the V-pits for example form sidewalls in the QW stack [32] which in principle should lead to diffuse scattering of the X-rays, it is unlikely that these can ascribe for the discrepancies we observed given that these samples have similar V-pit and trench defect densities, with densities of $4-5 \times 10^{8} \mathrm{~cm}^{-2}$ and $2-3 \times 10^{7} \mathrm{~cm}^{-2}$ respectively.

4.2 Roughness directionality In essence the scattering of the $\mathrm{X}$-rays by the interface is sensitive to the orientation of the interface. In the results presented so far, the samples were positioned on the diffractometer stage with the $[11 \overline{2} 0]$ direction perpendicular to the scattering plane. In this configuration, the incident X-rays come perpendicular to the changes in topography associated with the atomic steps and $\mathrm{InGaN}$ strips, which then gives rise to a detectable diffuse scattering. In principle, it would be possible to orientate the sample relative to the X-ray beam in order to intensify or attenuate the impact of specific aspects of the interface topography. In this section we rotate the sample around the azimuthal angle $\phi$ to obtain a more detailed picture of the interface topography.

Figure 6 present the results obtained for sample Q2T as well as for samples $2 \mathrm{~T} 0.25^{\circ}$ and $2 \mathrm{~T} 0.5^{\circ}$. For samples Q2T and $2 \mathrm{~T} 0.25^{\circ}$, very clear variations in roughness with azimuthal angle can be observed. This indicates that there is a preferred orientation of the roughening mechanisms in these samples, as expected from the presence of oriented troughs and steps. It can also be noted that the roughness of sample Q2T peaks at a level similar to sample $2 \mathrm{~T} 0.25^{\circ}$. This seems to supports the suggestion that the terraced structure of the QW before low temperature $\mathrm{GaN}$ growth gives rise to uneven indium incorporation in bar- 
rier leading to oriented roughness of the isoconcentration surface.

Surprisingly sample $2 \mathrm{~T} 0.5^{\circ}$ does not exhibit strong variations of roughness with $\phi$ despite being the sample with the most InGaN strips (and presumably the greatest roughness). However it should be emphasised that the scattering of X-rays at the interface is sensitive to the actual orientation of the variation in topography rather than on the orientation of the InGaN strips themselves, which may be somehow misleading. In the case of sample $2 \mathrm{~T} 0.25^{\circ}$, we can distinguish from Figure 4(d) that the strip edges are rather uniform and this uniformity of orientation leads to the increase in roughness with $\phi$ which we detected by XRR. On the other hand for sample $2 \mathrm{~T} 0.5^{\circ}$ miscut, Figure $4(\mathrm{~d})$ reveals that the edges of the $\mathrm{InGaN}$ strips are very jagged despite the strips being largely parallel to [1120], leading to a rough interface with no preferred orientation.

We attempted to recover the aforementioned results using the topography obtained by AFM. As mentioned earlier, the interface roughness contributes to the diffuse scattering (and hence to a detectable roughness) when the inclined topography scatters the incident beam in the plane defined by the X-ray source and the detector. Hence the critical component to consider is the projection of the topography gradient in the source-detector direction. We thus used the AFM scan presented in Figure 4(b),(d),(e) to extract the topography gradient $\vec{\nabla} z$ at each pixel. Because the gradient is very sensitive to sharps changes in height, the AFM scans were smoothed in order to remove instrument-induced sudden changes in topography, especially these occurring in the slow scan direction. The topography gradient was then projected onto a rotating unit vector $\overrightarrow{u_{\phi}}$ denoting the direction of the incident X-ray beam projected onto the (0001) plane. This axis is rotated by an angle $\phi$ with respect to the X-axis of the AFM scan, i.e. $\overrightarrow{u_{\phi}}\left(\phi=0^{\circ}\right)$ is perpendicular to $[11 \overline{2} 0]$. To obtain a description of the roughness as obtained by XRR we measured the standard deviation of the value $\vec{\nabla} z \cdot \overrightarrow{u_{\phi}}$ (note that these two values are expected to be comparable only on a relative scale).

Figure 7 presents the comparison between the roughness obtained by XRR and our model based on the gradient of AFM topography. It should be kept in mind the major difference here between the two techniques, which is that XRR provides a description of the average interface at the atomic scale for an illuminated area of several millimetres, while AFM can only achieve this precision (often limited by the tip status) with a field of view of the micrometre. Note also that a $\phi$ shift of up to $10^{\circ}$ to $20^{\circ}$ between the two curves is not surprising as it may result from the manual positioning of the wafer in the equipments. Taking this into consideration, the AFM model seems to mimic the trend in the XRR results. (Note that the shift in $\phi$ observed for $2 \mathrm{~T}$ $0.5^{\circ}$, is close to $45^{\circ}$, which cannot be explained by the sample positioning accuracy but perhaps as a consequence of differences of the jaggedness of the InGaN strip at the dif-

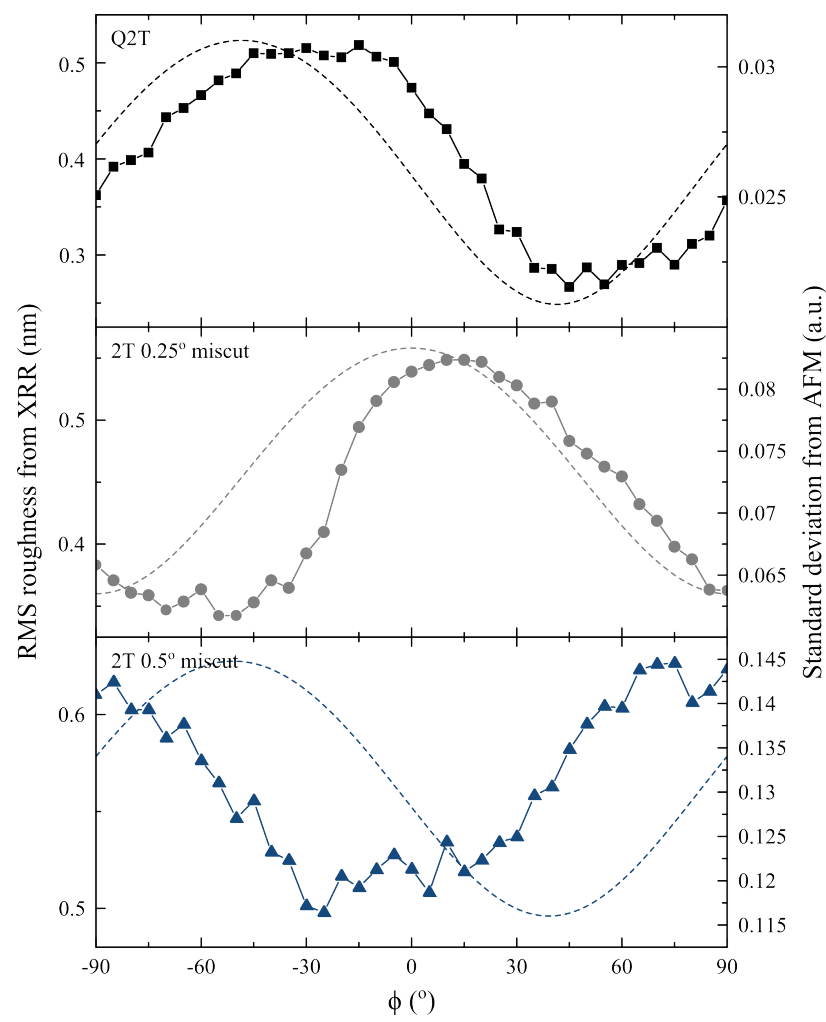

Figure 7 Comparison between the RMS roughness obtained by XRR (full line) and the standard deviation of the height gradient obtained from AFM scans (dotted line).

ferent length scale of the equipments.) Moreover while the RMS roughness varies with $\phi$ by $49 \%, 38 \%$ and $21 \%$ for samples Q2T, $2 \mathrm{~T} 0.25^{\circ}$ and $2 \mathrm{~T} 0.5^{\circ}$ respectively, the AFM model shows a similar trend, with values of 33\%, 24\% and $20 \%$ respectively. This confirms that there is a preferential direction of the roughening mechanism in samples Q2T, $2 \mathrm{~T} 0.25^{\circ}$ miscut as opposed to sample $2 \mathrm{~T} 0.5^{\circ}$ miscut.

5 Conclusion In conclusion, XRR was used to characterise the interface of InGaN/GaN QWs. The interface roughness was readily obtained from the ratio of diffuse to specular scatterings obtained on a transverse scan. The method allowed for quick identification of the presence or absence of GWWFs in the QW, as long as the interface is chemically sharp. By rotating the sample around the azimuthal angle, information about the directionality of the roughening mechanisms could be obtained. In the event where the interface is chemically graded, compositional non-uniformities across the terraced structure of the QW growth surface lead to aggravated roughness as the barrier is grown which may erroneously be described as GWWFs. Further analysis would be required to differentiate the two configurations.

This method could be well-suited for quick assessment of the QW interface when prior knowledge of the sample 
is already available. It might then be particularly useful to detect process deviation in a production line for example.

Acknowledgements This work has been funded in part by the EPSRC (under EP/H0495331) and the ERC (grant agreement no. 279361 (MACONS)).

\section{References}

[1] C. Humphreys, MRS Bulletin 33, 459 (2008).

[2] M. J. Galtrey, R. A. Oliver, M. J. Kappers, C. McAleese, D. Zhu, C. J. Humphreys, P. H. Clifton, D. Larson, and A. Cerezo, Applied Physics Letters 92(4), 041904 (2008).

[3] A. B. Yankovich, A. V. Kvit, X. Li, F. Zhang, V. Avrutin, H. Liu, N. Izyumskaya, Ü. Özgür, B. Van Leer, H. Morkoç, and P. M. Voyles, Microscopy and microanalysis 20(3), 864 (2014).

[4] N. K. Van der Laak， R. A. Oliver， M. J. Kappers, and C. J. Humphreys, Applied Physics Letters 90(12), 121911 (2007).

[5] R. A. Oliver, F. C-P. Massabuau, M. J. Kappers, W. A. Phillips, E. J. Thrush, C. C. Tartan, W.E. Blenkhorn, T. J. Badcock, P. Dawson, M. A. Hopkins, D. W. E. Allsopp, and C. J. Humphreys, Applied Physics Letters 103, 141114 (2013).

[6] G. H. Gu, D. H. Jang, K. B. Nam, and C. G. Park, Microscopy and microanalysis 19(August), 99 (2013).

[7] Z. Yang, R. Li, Q. Wei, T. Yu, Y. Zhang, W. Chen, and X. Hu, Applied Physics Letters 94(6), 061120 (2009).

[8] P. M. McBride, Q. Yan, and C. G. Van de Walle, Applied Physics Letters 105, 083507 (2014).

[9] L. Zhang, R. R. Lieten, M. Latkowska, M. Baranowski, R. Kudrawiec, K. Cheng, H. Liang, and G. Borghs, Japanese Journal of Applied Physics 52, 08JL10 (2013).

[10] F. C-P. Massabuau, M. J. Davies, W. E. Blenkhorn, S. Hammersley, M. J. Kappers, C. J. Humphreys, P. Dawson, and R. A. Oliver, Physica Status Solidi B 252(5), 928 (2015).

[11] T. Yan, J. He, W. Yang, K. Rajabi, W. Chen, J. Wu, X. Kang, G. Zhang, and X. Hu, Physica Status Solidi A 212(5), 925 (2015).

[12] R. Vaxenburg, E. Lifshitz, and A. .L Efros, Applied Physics Letters 102(3), 031120 (2013).

[13] H. Zhao, G. Liu, J. Zhang, J. D. Poplawsky, V. Dierolf, and N. Tansu, Optics express 19 Suppl 4, A991 (2011).

[14] T. Mehrtens, M. Schowalter, D. Tytko, P. Choi, D. Raabe, L. Hoffmann, H. Jönen, U. Rossow, A. Hangleiter, and A. Rosenauer, Applied Physics Letters 102(13), 132112 (2013).

[15] L. Hoffmann, H. Bremers, H. Jönen, U. Rossow, M. Schowalter, T. Mehrtens, A. Rosenauer, and A. Hangleiter, Applied Physics Letters 102, 102110 (2013).

[16] F. C-P. Massabuau, C.C. Tartan, R. Traynier, W.E. Blenkhorn, M. J. Kappers, P. Dawson, C. J. Humphreys, and R. A. Oliver, Journal of Crystal Growth 386, 88 (2014).

[17] T. M. Smeeton, M. J. Kappers, J. S. Barnard, M. E. Vickers, and C. J. Humphreys, Applied Physics Letters 83(26), 5419 (2003).

[18] K. H. Baloch, A.C. Johnston-Peck, K. Kisslinger, E. A. Stach, and S. Gradečak, Applied Physics Letters 102, 191910 (2013).
[19] D. Bowen and B. Tanner, X-ray metrology in semiconductor manufacturing (CRC/Taylor \& Francis, 2006).

[20] T. M. Smeeton, M. J. Kappers, J. S. Barnard, M. E. Vickers, and C. J. Humphreys, Phys. Status Solidi B 240(2), 297 (2003).

[21] M. J. Davies, P. Dawson, F. C-P. Massabuau, R. A. Oliver, M. J. Kappers, and C. J. Humphreys, Applied Physics Letters 105(9), 092106 (2014).

[22] S. Sintonen, T. Suihkonen, O. Svensk, P. T. Törmä, M. Ali, M. Sopanen, and H. Lipsanen, Physica Status Solidi C 7-8, 1790-1793 (2010).

[23] A. Krost, J. Bläsing, M. Lünenbürger, H. Protzmann, and M. Heuken, Applied Physics Letters 75, 689 (1999).

[24] M. Wormington, I. Pape, T. P. A. Hase, B. K. Tanner, and D. K. Bowen, Philosophical Magazine Letters 74(3), 2116 (1996).

[25] G. Ju, S. Fuchi, M. Tabuchi, and Y. Takeda, Journal of Applied Physics 114(12), 124906 (2013).

[26] G. Ju, Y. Honda, M. Tabuchi, Y. Takeda, and H. Amano, Journal of Applied Physics 115(9), 094906 (2014).

[27] S. K. Sinha, E. B. Sirota, S. Garoff, and H. B. Stanley, Physical Review B 38, 2297 (1988).

[28] R. D. Deslattes, E. G. Kessler Jr, P. Indelicato, and E. Lindroth, International Tables for Crystallography C (Berlin: Springer, 2006).

[29] N. Jouvet, M. J. Kappers, C. J. Humphreys, and R. A. Oliver, Journal of Applied Physics 113, 063503 (2013).

[30] N. Sharma, P. Thomas, D. Tricker, and C. Humphreys, Applied Physics Letters 77(9), 1274 (2000).

[31] F. C-P. Massabuau, S.-L. Sahonta, L. Trinh-Xuan, S. Rhode, T. J. Puchtler, M. J. Kappers, C. J. Humphreys, and R. A. Oliver, Applied Physics Letters 101, 212107 (2012).

[32] A. Hangleiter, F. Hitzel, C. Netzel, D. Fuhrmann, U. Rossow, G. Ade, and P. Hinze, Physical Review Letters 95(12), 127402 (2005). 